\title{
Synthesis and Reactions of New Thienopyridines, Pyridothienopyrimidines and Pyridothienotriazines
}

\author{
E. A. Bakhite," A. E. Abdel-Rahman, O. S. Mohamed, and E. A. Thabet \\ Chemistry Department, Factly of Science, Assint Cniversitv Assint 71516, Egypt \\ Recerved June 11, 2002
}

\begin{abstract}
4-Aryl-3-cyano-6-(2'-thienyl)-pyridine-2(1H)-thiones (1a-c) were reacted with phenacyl bromide chloro- $\mathrm{N}$ arylacetamides or 2-chloroacetylaminopyridine to funish 2 -functionalized 3-amino-4-aryl-6-(2'-thienyl)theno[2,3-b]pyridines 4a-c and 5a-c or $\mathbf{5} \mathbf{d}$ respectively. The conpounds $\mathbf{5 a - d}$ undenvent different sequence of reactions to produce a variety of thienylpyridothienopy rimidines and. thienylpyridothienotriazines. Some of the prepared compounds were tested in vitro for their antimicrobial activities.
\end{abstract}

Key Words : Thienopyridines. Pỵrimidines. Triazines

\section{Introduction}

The biological activities of condensed pyrimidines as sedatives. antibacterials and antimalarials are well documented. ${ }^{1.2}$ Several thieno[2.3-b]pyridine derivatives are known to possess antibacterial ${ }^{3-5}$ antilyypertensive ${ }^{6}$ and gonadotropinreleasing hormone antagonizing ${ }^{7.8}$ activity. Pyridothienopyrimidine derivatives have been found applications as analgesics. antipyretics ${ }^{10}$ and anti-inflammatories. ${ }^{11}$ Moreover. some pyridothienotriazines are known to exhibit<smiles>N#Cc1c(Br)cc(-c2cccs2)nc1SCC(=O)O</smiles><smiles>Nc1c(C(=O)c2ccccc2)sc2nc(-c3cccs3)cc([Al])c12</smiles><smiles>[3H]I</smiles><smiles>N#Cc1c(Br)cc(-c2cccs2)[nH]c1=S</smiles>
1a-c antianaphylactic..$^{12}$ and antiallergic activity..$^{13}$ In view of these facts and as a continuation of our previous work on thieno[2.3-b]pyridines. ${ }^{1417}$ we report herein the synthesis of the title compounds and their evaluation regarding antimicrobial activities.

\section{Results and Discussion}

Based on the important synthetic utility of many 3cyanopyridine-2( $(H)$-thione derivatives. ${ }^{12-17}$ the 4-aryl-3-

\begin{tabular}{c|l}
\hline $\mathbf{1 , 2 , 4}$ & $\mathrm{Ar}$ \\
\hline $\mathrm{a}$ & $\mathrm{C}_{6} \mathrm{H}_{5}$ \\
b & $4-\mathrm{CH}_{3} \mathrm{OC}_{6} \mathrm{H}_{4}$ \\
c & $4-\mathrm{ClC}_{6} \mathrm{H}_{4}$
\end{tabular}
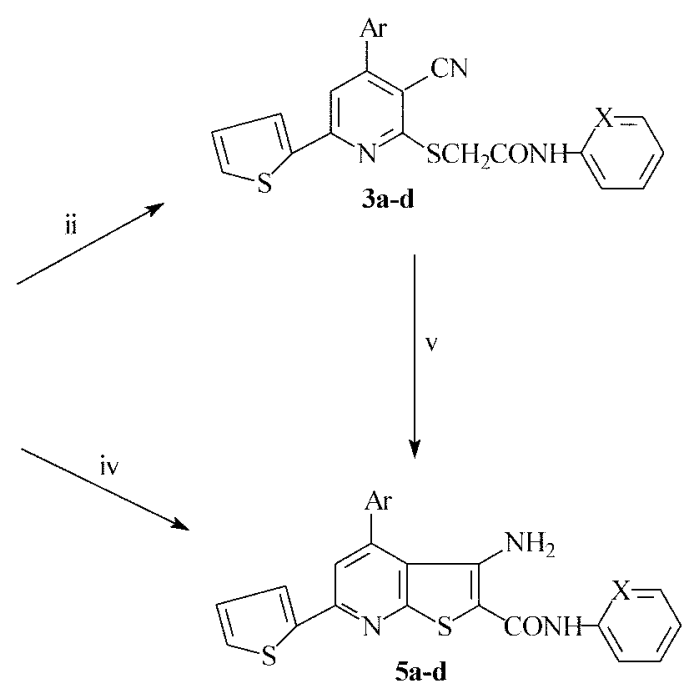

\begin{tabular}{c|l|c}
\hline $\mathbf{3 , 5}$ & \multicolumn{1}{|c|}{$\mathrm{Ar}$} & $\mathrm{X}$ \\
\hline $\mathrm{a}$ & $\mathrm{C}_{6} \mathrm{H}_{5}$ & $\mathrm{CH}$ \\
$\mathrm{b}$ & $4-\mathrm{CH}_{3} \mathrm{OC}_{6} \mathrm{H}_{4}$ & $\mathrm{CH}$ \\
$\mathrm{c}$ & $4-\mathrm{ClC}_{6} \mathrm{H}_{4}$ & $\mathrm{CH}$ \\
$\mathrm{d}$ & $\mathrm{C}_{6} \mathrm{H}_{5}$ & $\mathrm{~N}$
\end{tabular}

i: $\mathrm{PhCOCH}_{2} \mathrm{Br} / \mathrm{AcONa}$; ii: $\mathrm{PhCOCH}_{2} \mathrm{Br} / \mathrm{EtONa}$ iii: $\mathrm{ClCH}_{2} \mathrm{CONH} / \mathrm{AcONa}$; iv: $\mathrm{ClCH}_{2} \mathrm{CONH}$

Scheme 1

Corresponding Author. e-mail: etiafỵâtaun.eun.eg 
cyano-6-( $2^{\prime}$-thienyl)-pyridine-2(1H)-thiones (1a-c) were used as starting materials in the synthesis of the target heterocycles. The reaction of 1a-c with some halocompounds namely: phenacyl bromide. chloro- $N$-phenylacetamide or 2chloroacetylaminopyridine in the presence of sodium acetate trihy'drate gave 2-substituted methylthio-4-aryl-3-cyano-6(2'-thienyl)-pyridines (2a-c $\mathbf{3 a - c}$ and $3 \mathrm{~d}$ ). Upon treatment of these compounds with sodium ethoxide in ethanol ther underwent intramolecular Thorpe-Ziegler cyclization to furnish 2-functionalized 3-amino-4-aryl-6-(2'-thienyl)-thieno[2.3-b]pyridines (4a-c. 5a-c and 5d). The latter thienopyridine derivatives were also prepared via direct reaction of 1a-c with the respective halocompounds in the presence of sodium ethoxide as a basic catalyst (Scheme 1).

The cyclocondensation of 5a-d with triethyl orthoformate in the presence of acetic anhydride afforded pyrido[3'. $2^{\prime}$ : 4.5]thieno[3.2-d]pyrimidine-4(3H)-ones 6a-d. The triazinone analogs $7 \mathbf{a}-\mathbf{d}$ were prepared by diazotisation of $\mathbf{5 a - d}$ using sodium nitrite and acetic acid-sulfuric acid mixture. The interaction of 5a-d with carbon disulfide in hot pyridine resulted in the formation of thioxopyrimidinone derivatives 8a-d (Scheme 2).

The reaction of $\mathbf{8 a}$ with methyl iodide in ethanolic sodium hy̆droxide solution gave 3.9-diphenỵl-2-methylthio-7-(2'- thienyl)-pyrido[3', 2':4.5]thieno[3.2-d]pyrimidine-4(3H)-one (9). Compound 10 was synthesized through the reaction of $\mathbf{8 a}$ with phenacyl bromide in the presence of sodium acetate. Heating 8a with hydrazine hydrate in pyridine resulted in the formation of hydrazinopyrimidinone derivative 11 which underwent a cyclocondensation reaction upon heating with triethyl orthoformate under neat conditions to furnish 4.10 diphenyl-8-(2'-thienyl)-s-triazolo[4". $3^{\prime \prime}$-a]py rido[3'.2':4.5]thieno[2,3-e]pyrimidine-5( $4 H)$-one (12) (Scheme 3 ).

The structural formulas of all newly sytnthesized compounds were established and confirmed on the basis of their elemental analyses and spectral data (cf. Tables 1. 2 and experimental part).

Some of the synthesized compounds were evaluated $\mathrm{in}$ witro for their antimicrobial activities against three strains of bacteria and two fungal species (Table 3) using filter paper disc diffusion method. ${ }^{18}$ Tioconazole (Tyrosyde ${ }^{-2}$ (13) which contains a thiophene moiety in its structure was used as a reference substance. The results indicated that most of the tested compounds exhibited moderate to strong activities agaisnt Staphylococcus atrets, Bacillus certis and Serratia rhodnii. However. concerning the antifungal activity: all tested compounds showed no effects except for compounds 3a. 3d. 6a, 7d. 9 and 12 which showed cosiderable activity<smiles>O=c1c2sc3nc(-c4cccs4)cc([Al])c3c2ncn1-c1ccccc1</smiles><smiles>[3H][CH]</smiles>

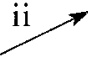<smiles>O=c1c2sc3nc(-c4cccs4)cc([Al])c3c2nnn1-c1[X]cccc1</smiles>

5a-d<smiles>[Y19]c1cc(-c2cccs2)nc2sc3c(=O)n(-c4[X]cccc4)c(=S)[nH]c3c12</smiles><smiles>C[14CH3]</smiles>

\begin{tabular}{c|l|l}
\hline $6-8$ & \multicolumn{1}{|c|}{$\mathrm{Ar}$} & $X$ \\
\hline$a$ & $\mathrm{C}_{6} \mathrm{H}_{5}$ & $\mathrm{CH}$ \\
6 & $4-\mathrm{CH}_{3} \mathrm{OC}_{6} \mathrm{H}_{4}$ & $\mathrm{CH}$ \\
$c$ & $4-\mathrm{CK}_{6} \mathrm{H}_{4}$ & $\mathrm{CH}$ \\
$d$ & $\mathrm{C}_{6} \mathrm{H}_{5}$ & $\mathrm{~N}$
\end{tabular}

i: $\mathrm{HC}(\mathrm{OEt})_{3} / \mathrm{Ac}_{2} \mathrm{O}$; ii: $\mathrm{NaNO}_{2} / \mathrm{H}_{2} \mathrm{SO}_{4}$ - $\mathrm{ACOH}$; iii: $\mathrm{CS}_{2} /$ pyridine

Scheme 2<smiles>CC(C)c1nc2c(sc3nc(-c4cccs4)cc(-c4ccccc4)c32)c(=O)n1-c1ccccc1</smiles><smiles>CC(C)C</smiles>

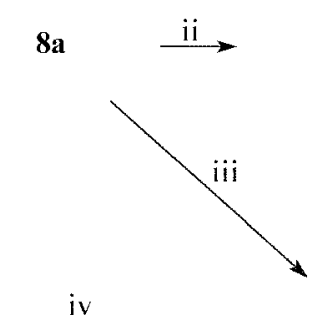<smiles>O=C(O)CSc1nc2c(sc3nc(-c4cccs4)cc(-c4ccccc4)c32)c(=O)n1-c1ccccc1</smiles><smiles>NNc1nc2c(sc3nc(-c4cccs4)cc(-c4ccccc4)c32)c(=O)n1-c1ccccc1</smiles>

i: $\mathrm{CH}_{3} \mathrm{I} / \mathrm{NaOH}$; ii: $\mathrm{PhCOCH}_{2} \mathrm{Br} / \mathrm{AcONa}$; iii: $\mathrm{N}_{2} \mathrm{H}_{4} \cdot \mathrm{H}_{2} \mathrm{O}$; iv: $\mathrm{CH}(\mathrm{OEt})_{3}$

Scheme 3 
Table 1. Melting points, vields and analvical data of the syinthesized compounds

\begin{tabular}{|c|c|c|c|c|c|c|c|}
\hline \multirow{2}{*}{ Compd. } & \multirow{2}{*}{$\begin{array}{l}\text { M.p. }{ }^{\circ} \mathrm{C} \\
\text { yield, } \%\end{array}$} & \multirow{2}{*}{$\begin{array}{l}\text { Fonmula } \\
\text { (M.W.) }\end{array}$} & \multicolumn{5}{|c|}{ Calculated / Found } \\
\hline & & & $\% \mathrm{C}$ & $\% \mathrm{H}$ & $\% \mathrm{~N}$ & $\% \mathrm{~S}$ & $\% \mathrm{Cl}$ \\
\hline $2 a$ & $\begin{array}{c}170 \\
90\end{array}$ & $\begin{array}{c}\mathrm{C}_{24} \mathrm{H}_{16} \mathrm{~N}_{2} \mathrm{OS}_{2} \\
(412.54)\end{array}$ & $\begin{array}{l}69.88 \\
69.91\end{array}$ & $\begin{array}{l}3.91 \\
3.85\end{array}$ & $\begin{array}{l}6.79 \\
6.74\end{array}$ & $\begin{array}{l}15.54 \\
15.29\end{array}$ & \\
\hline $2 b$ & $\begin{array}{c}165 \\
91\end{array}$ & $\begin{array}{c}\mathrm{C}_{25} \mathrm{H}_{18} \mathrm{~N}_{2} \mathrm{O}_{2} \mathrm{~S}_{2} \\
(4+42.55)\end{array}$ & $\begin{array}{l}67.85 \\
67.67\end{array}$ & $\begin{array}{l}4.09 \\
4.14\end{array}$ & $\begin{array}{l}6.33 \\
6.51\end{array}$ & $\begin{array}{l}19.49 \\
14.19\end{array}$ & \\
\hline $2 c$ & $\begin{array}{c}160 \\
90\end{array}$ & $\begin{array}{c}\mathrm{C}_{\Theta_{4} \mathrm{H}_{1} \leqslant \mathrm{CIN}_{2} \mathrm{OS}_{2}} \\
(446.97)\end{array}$ & $\begin{array}{l}64.49 \\
64.62\end{array}$ & $\begin{array}{l}3.38 \\
3.51\end{array}$ & $\begin{array}{l}6.27 \\
6.34\end{array}$ & $\begin{array}{l}14.35 \\
14.49\end{array}$ & $\begin{array}{l}7.93 \\
7.82\end{array}$ \\
\hline $3 a$ & $\begin{array}{c}200 \\
94\end{array}$ & $\begin{array}{c}\mathrm{C}_{24} \mathrm{H}_{1}-\mathrm{N}_{3} \mathrm{OS} \\
(427.55)\end{array}$ & $\begin{array}{l}67.42 \\
67.55\end{array}$ & $\begin{array}{l}4.01 \\
4.08\end{array}$ & $\begin{array}{l}9.83 \\
9.69\end{array}$ & $\begin{array}{l}14.10 \\
14.01\end{array}$ & \\
\hline $3 b$ & $\begin{array}{c}210 \\
95\end{array}$ & $\begin{array}{c}\mathrm{C}_{2} \leqslant \mathrm{H}_{14} \mathrm{~N}_{3} \mathrm{O}_{2} \mathrm{~S}_{2} \\
(457.57)\end{array}$ & $\begin{array}{l}65.62 \\
65.59\end{array}$ & $\begin{array}{l}4.19 \\
4.00\end{array}$ & $\begin{array}{l}9.18 \\
9.07\end{array}$ & $\begin{array}{l}14.02 \\
14.32\end{array}$ & \\
\hline $3 c$ & $\begin{array}{c}210 \\
92\end{array}$ & $\begin{array}{c}\mathrm{C}_{24} \mathrm{H}_{1 \mathrm{n}} \mathrm{ClN}_{3} \mathrm{OS}_{2} \\
(462.00)\end{array}$ & $\begin{array}{l}62.40 \\
62.64\end{array}$ & $\begin{array}{l}3.49 \\
3.62\end{array}$ & $\begin{array}{l}9.09 \\
9.39\end{array}$ & $\begin{array}{l}13.88 \\
14.03\end{array}$ & $\begin{array}{l}7.68 \\
7.90\end{array}$ \\
\hline $3 d$ & $\begin{array}{l}190 \\
85\end{array}$ & $\begin{array}{c}\mathrm{C}_{23} \mathrm{H}_{1 \leqslant} \mathrm{~N}_{4} \mathrm{OS} \\
(428.54)\end{array}$ & $\begin{array}{l}64.47 \\
64.65\end{array}$ & $\begin{array}{l}3.76 \\
3.86\end{array}$ & $\begin{array}{l}13.07 \\
13.03\end{array}$ & $\begin{array}{l}14.96 \\
15.01\end{array}$ & \\
\hline $4 a$ & $\begin{array}{l}180 \\
85\end{array}$ & $\begin{array}{c}\mathrm{C}_{24} \mathrm{H}_{1} \mathrm{~N}_{2} \mathrm{OS} \\
(412.54)\end{array}$ & $\begin{array}{l}69.88 \\
69.30\end{array}$ & $\begin{array}{l}3.91 \\
3.75\end{array}$ & $\begin{array}{l}6.79 \\
6.92\end{array}$ & $\begin{array}{l}15.54 \\
15.74\end{array}$ & \\
\hline th & $\begin{array}{l}192 \\
87\end{array}$ & $\begin{array}{c}\mathrm{C}_{25} \mathrm{H}_{18} \mathrm{~N}_{2} \mathrm{O}_{2} \mathrm{~S}_{2} \\
(4+42.55)\end{array}$ & $\begin{array}{l}67.85 \\
67.79\end{array}$ & $\begin{array}{l}4.09 \\
4.04\end{array}$ & $\begin{array}{l}6.33 \\
6.52\end{array}$ & $\begin{array}{l}14.49 \\
14.61\end{array}$ & \\
\hline $4 c$ & $\begin{array}{c}275 \\
87\end{array}$ & $\begin{array}{c}\mathrm{C}_{2+} \mathrm{H}_{13} \mathrm{ClN}_{2} \mathrm{OS}_{2} \\
(446.97)\end{array}$ & $\begin{array}{l}64.49 \\
64.69\end{array}$ & $\begin{array}{l}3.38 \\
3.53\end{array}$ & $\begin{array}{l}6.27 \\
6.12\end{array}$ & $\begin{array}{l}14.35 \\
14.40\end{array}$ & $\begin{array}{l}7.93 \\
7.95\end{array}$ \\
\hline $5 \mathbf{a}$ & $\begin{array}{c}235 \\
88\end{array}$ & $\begin{array}{c}\mathrm{C}_{24} \mathrm{H}_{1: \mathrm{N}_{3} \mathrm{OS}} \\
(427.55)\end{array}$ & $\begin{array}{l}67.42 \\
67.35\end{array}$ & $\begin{array}{l}4.01 \\
4.14\end{array}$ & $\begin{array}{l}9.83 \\
9.90\end{array}$ & $\begin{array}{l}14.10 \\
14.12\end{array}$ & \\
\hline $5 b$ & $\begin{array}{c}220 \\
85\end{array}$ & $\begin{array}{c}\mathrm{C}_{25} \mathrm{H}_{10 \mathrm{~N}_{3} \mathrm{O}_{2} \mathrm{~S}_{2}}^{(457.57)}\end{array}$ & $\begin{array}{l}65.62 \\
65.86\end{array}$ & $\begin{array}{l}4.19 \\
4.34\end{array}$ & $\begin{array}{l}9.18 \\
9.31\end{array}$ & $\begin{array}{l}14.02 \\
14.00\end{array}$ & \\
\hline $5 c$ & $\begin{array}{c}240 \\
84\end{array}$ & $\begin{array}{c}\mathrm{C}_{2+} \mathrm{H}_{16} \mathrm{CIN}_{3} \mathrm{OS}_{2} \\
(462.00)\end{array}$ & $\begin{array}{l}62.40 \\
62.56\end{array}$ & $\begin{array}{l}3.49 \\
3.65\end{array}$ & $\begin{array}{l}9.09 \\
9.03\end{array}$ & $\begin{array}{l}13.88 \\
13.65\end{array}$ & $\begin{array}{l}7.68 \\
7.70\end{array}$ \\
\hline $5 d$ & $\begin{array}{c}130 \\
80\end{array}$ & $\begin{array}{c}\mathrm{C}_{23} \mathrm{H}_{15} \mathrm{~N}_{4} \mathrm{OS} \mathrm{S}_{2} \\
(428.54)\end{array}$ & $\begin{array}{l}64.47 \\
64.12\end{array}$ & $\begin{array}{l}3.76 \\
3.50\end{array}$ & $\begin{array}{l}13.07 \\
13.00\end{array}$ & $\begin{array}{l}14.96 \\
14.85\end{array}$ & \\
\hline $6 a$ & $\begin{array}{l}330 \\
95\end{array}$ & $\begin{array}{c}\mathrm{C}_{2} \leqslant \mathrm{H}_{1}: \mathrm{N}_{3} \mathrm{OS} \\
(437.55)\end{array}$ & $\begin{array}{l}68.63 \\
68.43\end{array}$ & $\begin{array}{l}3.46 \\
3.34\end{array}$ & $\begin{array}{l}9.60 \\
9.55\end{array}$ & $\begin{array}{l}14.66 \\
14.72\end{array}$ & \\
\hline $6 \mathrm{~b}$ & $\begin{array}{c}190 \\
90\end{array}$ & $\begin{array}{c}\mathrm{C}_{20} \mathrm{H}_{1}-\mathrm{N}_{3} \mathrm{O}_{2} \mathrm{~S}_{2} \\
(467.56)\end{array}$ & $\begin{array}{l}66.79 \\
66.88\end{array}$ & $\begin{array}{l}3.66 \\
3.34\end{array}$ & $\begin{array}{l}8.99 \\
8.90\end{array}$ & $\begin{array}{l}13.72 \\
13.79\end{array}$ & \\
\hline $6 \mathrm{c}$ & $\begin{array}{c}270 \\
92\end{array}$ & $\begin{array}{c}\mathrm{C}_{4} \mathrm{H}_{14} \mathrm{ClN}_{3} \mathrm{OS}_{2} \\
(471.98)\end{array}$ & $\begin{array}{l}63.62 \\
63.42\end{array}$ & $\begin{array}{l}2.99 \\
3.01\end{array}$ & $\begin{array}{l}8.90 \\
8.99\end{array}$ & $\begin{array}{l}13.59 \\
13.79\end{array}$ & $\begin{array}{l}7.51 \\
7.70\end{array}$ \\
\hline $6 \mathrm{~d}$ & $\begin{array}{l}325 \\
90\end{array}$ & $\begin{array}{c}\mathrm{C}_{24} \mathrm{H}_{14} \mathrm{~N}_{4} \mathrm{OS} \\
(438.53)\end{array}$ & $\begin{array}{l}65.73 \\
65.63\end{array}$ & $\begin{array}{l}3.22 \\
3.14\end{array}$ & $\begin{array}{l}12.78 \\
12.50\end{array}$ & $\begin{array}{l}14.62 \\
14.40\end{array}$ & \\
\hline $7 a$ & $\begin{array}{l}300 \\
92\end{array}$ & $\begin{array}{c}\mathrm{C}_{24} \mathrm{H}_{14} \mathrm{~N}_{4} \mathrm{OS} \\
(438.53)\end{array}$ & $\begin{array}{l}65.73 \\
65.59\end{array}$ & $\begin{array}{l}3.22 \\
3.13\end{array}$ & $\begin{array}{l}12.78 \\
12.70\end{array}$ & $\begin{array}{l}14.62 \\
14.68\end{array}$ & \\
\hline $7 b$ & $\begin{array}{c}285 \\
90\end{array}$ & $\begin{array}{c}\mathrm{C}_{2}<\mathrm{H}_{1 \times} \mathrm{N}_{4} \mathrm{O}_{2} \mathrm{~S}_{2} \\
(468.55)\end{array}$ & $\begin{array}{l}64.08 \\
64.32\end{array}$ & $\begin{array}{l}3.44 \\
3.30\end{array}$ & $\begin{array}{l}11.96 \\
12.02\end{array}$ & $\begin{array}{l}13.69 \\
13.90\end{array}$ & \\
\hline $7 \mathrm{c}$ & $\begin{array}{c}270 \\
90\end{array}$ & $\begin{array}{c}\mathrm{C}_{\lambda_{4}} \mathrm{H}_{13} \mathrm{CIN}_{4} \mathrm{OS}_{2} \\
(472.97)\end{array}$ & $\begin{array}{l}60.95 \\
61.02\end{array}$ & $\begin{array}{l}2.77 \\
2.90\end{array}$ & $\begin{array}{l}11.85 \\
11.91\end{array}$ & $\begin{array}{l}13.56 \\
13.15\end{array}$ & $\begin{array}{l}7.50 \\
7.74\end{array}$ \\
\hline $7 \mathrm{~d}$ & $\begin{array}{c}280 \\
90\end{array}$ & $\begin{array}{c}\mathrm{C}_{23} \mathrm{H}_{12} \mathrm{~N}_{5} \mathrm{OS}_{2} \\
(439.52)\end{array}$ & $\begin{array}{l}62.85 \\
62.90\end{array}$ & $\begin{array}{l}2.98 \\
2.85\end{array}$ & $\begin{array}{l}15.93 \\
15.75\end{array}$ & $\begin{array}{l}14.59 \\
14.33\end{array}$ & \\
\hline $8 a$ & $\begin{array}{c}>360 \\
71\end{array}$ & $\begin{array}{c}\mathrm{C}_{25} \mathrm{H}_{15} \mathrm{~N}_{3} \mathrm{OS}_{3} \\
(469.61)\end{array}$ & $\begin{array}{l}63.94 \\
63.75\end{array}$ & $\begin{array}{l}3.22 \\
3.18\end{array}$ & $\begin{array}{l}8.95 \\
8.83\end{array}$ & $\begin{array}{l}20.48 \\
20.61\end{array}$ & \\
\hline $8 b$ & $\begin{array}{c}280 \\
70\end{array}$ & $\begin{array}{c}\mathrm{C}_{26} \mathrm{H}_{1}: \mathrm{N}_{3} \mathrm{O}_{2} \mathrm{~S}_{3} \\
(499.63)\end{array}$ & $\begin{array}{l}62.50 \\
62.75\end{array}$ & $\begin{array}{l}3.43 \\
3.29\end{array}$ & $\begin{array}{l}8.41 \\
8.34\end{array}$ & $\begin{array}{l}19.25 \\
19.11\end{array}$ & \\
\hline $8 c$ & $\begin{array}{c}345 \\
66\end{array}$ & $\begin{array}{c}\mathrm{C}_{25} \mathrm{H}_{14} \mathrm{CIN}_{3} \mathrm{OS}_{3} \\
(504.04)\end{array}$ & $\begin{array}{l}59.57 \\
59.41\end{array}$ & $\begin{array}{l}2.80 \\
2.87\end{array}$ & $\begin{array}{l}8.34 \\
8.18\end{array}$ & $\begin{array}{l}19.09 \\
19.02\end{array}$ & $\begin{array}{l}7.03 \\
6.80\end{array}$ \\
\hline $8 d$ & $\begin{array}{l}315 \\
71\end{array}$ & $\begin{array}{c}\mathrm{C}_{24} \mathrm{H}_{14} \mathrm{~N}_{4} \mathrm{OS}_{3} \\
(470.60)\end{array}$ & $\begin{array}{l}61.25 \\
61.12\end{array}$ & $\begin{array}{l}2.99 \\
3.16\end{array}$ & $\begin{array}{l}11.91 \\
11.78\end{array}$ & $\begin{array}{l}20.44 \\
20.63\end{array}$ & \\
\hline 9 & $\begin{array}{l}335 \\
97\end{array}$ & 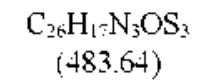 & $\begin{array}{l}64.57 \\
64.65\end{array}$ & $\begin{array}{l}3.54 \\
3.36\end{array}$ & $\begin{array}{l}8.69 \\
8.49\end{array}$ & $\begin{array}{l}19.89 \\
19.71\end{array}$ & \\
\hline
\end{tabular}

Table 1. Continued

\begin{tabular}{|c|c|c|c|c|c|c|c|}
\hline \multirow{2}{*}{ Compd. } & \multirow{2}{*}{$\begin{array}{l}\text { M.p., }{ }^{\circ} \mathrm{C} \\
\text { yield, } \%\end{array}$} & \multirow{2}{*}{$\begin{array}{l}\text { Fonmula } \\
\text { (M.W.) }\end{array}$} & \multicolumn{5}{|c|}{ Calculated / Found } \\
\hline & & & $\% \mathrm{C}$ & $\% \mathrm{H}$ & $\% \mathrm{~N}$ & $\% \mathrm{~S}$ & $\% \mathrm{Cl}$ \\
\hline \multirow[t]{2}{*}{9} & 335 & $\mathrm{C}_{2} \mathrm{H}_{1}: \mathrm{N}_{3} \mathrm{OS}_{3}$ & 64.57 & 3.54 & 8.69 & 19.89 & \\
\hline & 97 & $(483.64)$ & 64.65 & 3.36 & 8.49 & 19.71 & \\
\hline \multirow[t]{2}{*}{10} & 265 & $\mathrm{C}_{33} \mathrm{H}_{21} \mathrm{~N}_{3} \mathrm{O}_{2} \mathrm{~S}_{2}$ & 67.44 & 3.60 & 7.15 & 16.37 & \\
\hline & 80 & $(587.75)$ & 67.65 & 3.43 & 7.00 & 1602 & \\
\hline \multirow[t]{2}{*}{11} & 327 & $\mathrm{C}_{23} \mathrm{H}_{18} \mathrm{~N}_{5} \mathrm{OS}_{2}$ & 64.08 & 3.87 & 14.95 & 13,69 & \\
\hline & 47 & $(468.58)$ & 64.13 & 3.68 & 14.86 & 13.53 & \\
\hline \multirow[t]{2}{*}{12} & 285 & $\mathrm{C}_{24} \mathrm{H}_{14} \mathrm{~N}_{4} \mathrm{OS}_{2}$ & 65.39 & 3.17 & 14.66 & 13,43 & \\
\hline & 55 & $(477.57)$ & 65.12 & 3.01 & 14.42 & 13.35 & \\
\hline
\end{tabular}

against penicillitm oxalictm and /or Aspergillus fumigatus.<smiles>Clc1ccc(C(Cn2ccnc2)OCc2ccsc2Cl)c(Cl)c1</smiles>

\section{Experimental Section}

All melting points are uncorrected and measured on a Gallan-Kamp apparatus. IR spectra: Shimadzu 470 IRspectrophotometer $\left(\mathrm{KBr}\right.$ : $v_{\text {max }}$ in $\mathrm{cm}^{-1}$ ). ${ }^{1} \mathrm{H}$ NMR spectra: Varian EM-390, $90 \mathrm{MHz}{ }^{1} \mathrm{H}$ NMR spectrometer using TMS as intemal standard ( $\delta$ in ppm); MS: Jeol JMS-600; elemental analyses: Elementar Analy sensystem GmbH VARIOEL V2.3 July 1998 CHNS Mode: their results were in good agreement with the calculated values. Melting points, yields and analytical data of all newly synthesized compounds are listed in Table 1.

4-Aryl-3-cyano-6-(2'-thienyl)-pyridine-2(1H)-thiones (1ac). These compounds were prepared in analogy to the method described before. ${ }^{7}$

Reaction of 1 a-c with phenacyl bromide, chloro- $N$ phenylacetamide or 2-chloroacetylaminopyridine: Formation of $S$-substituted thiopyridines $2 a-c$ and $3 a-c$ or $3 d$. To a suspension of compound $1 \mathrm{a}-\mathrm{c}(20 \mathrm{mmol})$ and sodium acetate trihydrate $(3.0 \mathrm{~g} .22 \mathrm{mmol})$ in ethanol $(50$ $\mathrm{mL}$ ), the respective halocompound $(20 \mathrm{~mm}$ ol) was added. The resulting mixture was refluxed for 2 luss. The precipitate that formed on cooling was collected and recry stallized from ethanol as pale yellow needles of $\mathbf{2 a - c . ~ 3 a - c}$ or $\mathbf{3 d}$ respectively. (Tables 1,2)

2-Functionalized 3-amino-4-aryl-6-(2'-thienyl)-thieno[2,3-b]pyridines 4a-c and 5 a-d

Method A: Compounds $2 a-c$ or $3 a-d ~(10 \mathrm{mmol})$ were suspended in sodium ethoxide solution ( 0.12 g sodium in 30 $\mathrm{mIL}$ abs. ethanol) and heated under reflux for 5 mins. The solid that formed while hot was collected and recrystallized from ethanol-chloroform mixture to give canary yellow 
Table 2. IR, ${ }^{1} \mathrm{H}$ NMR and MS spectral data of compounds 2a-8d

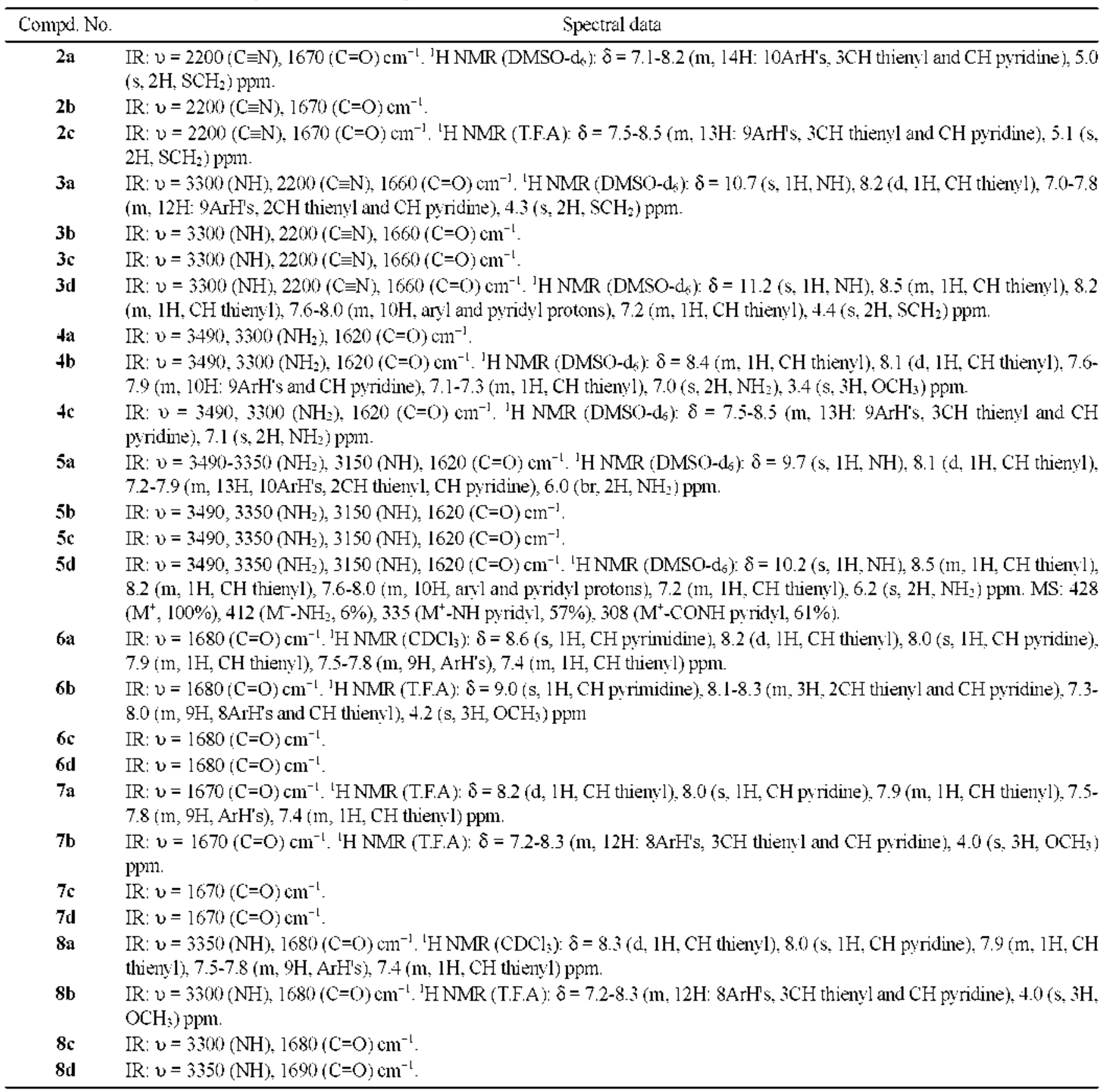

crystals of 4a-c and 7a-d respectively (Tables 1.2).

Method B: To a suspension of compound 1a-c (10 mumol) in sodium ethoxide solution $(0.35 \mathrm{~g}$ sodium in $40 \mathrm{~mL}$ abs. ethanol). the respective halocompound (10 $\mathrm{mmol})$ was added. The resulting mixture was refluxed for 20 mins. The formed yellow precipitate was collected and recrystallized from ethanol-chloroform mixture to give compounds ta-c and $\mathbf{5 a - d}$ in $76-82 \%$ yield. These products were identical in all aspects to those described in method $\mathrm{A}$.

Pyrido[3',2':4,5]thieno[3,2-d]pyrimidne- $-4(3 H)$-one derivatives 6a-d. A mixture of $\mathbf{5 a - d}(5 \mathrm{mmol})$ and triethyl orthoformate $(2 \mathrm{~mL})$ in acetic anhydride $(20 \mathrm{~mL})$ was refluxed for 4 hrs. The solid thus formed while hot was collected and recrystallized from ethanol-chloroform mixture to give white cry stals of 6a-d (Tables 1.2)

Pyrido[3',2':4,5]thieno[3,2-cl][1,2,3]triazine-4(3H)-one derivatives $7 \mathrm{a}$-d. Sodium nitrite solution $10 \%(5 \mathrm{~mL})$ was added to a solution of compound $5 \mathbf{a}-\mathrm{d}$ ( $2 \mathrm{mmol})$ in concentrated sulphuric acid $(5 \mathrm{~mL})$ and glacial acetic acid (5 $\mathrm{mL}$ ) at $0{ }^{\circ} \mathrm{C}$ during $5 \mathrm{mins}$. with stirring. The mixture was allowed to stand at room temperature for 30 mins. The solid that precipitated on dilution with water was collected and recrystallized from ethanol as white needles of $7 \mathbf{a}-\mathbf{d}$ (Tables 1. 2).

4-0x0-1,2,3,4-tetrahydro-2-thioxopyrido[3',2':4,5]thieno$[3,2-d]$ pyrimidine- $+(3 H)$-one derivatives $8 \mathrm{a}-\mathrm{d}$. A mixture 
Table 3. The antimicrobial activities of some representative compounds

\begin{tabular}{|c|c|c|c|c|c|}
\hline $\begin{array}{l}\text { Compd. } \\
\text { No. }\end{array}$ & $\begin{array}{c}\text { Staphylococcus } \\
\text { aurens }\end{array}$ & $\begin{array}{c}\text { Bacillus } \\
\text { certus }\end{array}$ & Serntia hodenii & $\begin{array}{l}\text { Pencillium } \\
\text { oxalicum }\end{array}$ & $\begin{array}{l}\text { Aspergillus } \\
\text { fumigatus }\end{array}$ \\
\hline $2 c$ & + & - & - & - & - \\
\hline $3 \mathbf{a}$ & - & ++ & + & - & + \\
\hline $3 d$ & + & - & ++ & + & + \\
\hline $4 c$ & ++ & ++ & - & - & - \\
\hline $5 a$ & + & + & + & - & - \\
\hline 5d & - & + & + & - & - \\
\hline $6 a$ & + & _ & - & + & + \\
\hline $6 c$ & ++ & $\overline{+}$ & + & - & - \\
\hline $7 b$ & - & ++ & ++ & - & - \\
\hline $7 d$ & + & - & - & ++ & - \\
\hline $8 \mathbf{a}$ & ++ & + & - & - & - \\
\hline $8 b$ & + & ++ & ++ & - & - \\
\hline 9 & ++ & + & ++ & + & + \\
\hline 10 & ++ & + & - & - & - \\
\hline 12 & + & ++ & + & ++ & + \\
\hline Tyrosyde & + & + & + & ++ & +++ \\
\hline
\end{tabular}

-: No activity: + : moderate activity (inhibition zone: $5-10 \mathrm{~mm}$ ); -+: strong activity (inhibition zone:11-15 mm): +++: fery strong activity (inhibition zone: $16-20 \mathrm{~mm}$ ).

of 5a-d $(3 \mathrm{mmol})$ and carbon disulphide $(3 \mathrm{~mL})$ in dry pyridine $(25 \mathrm{~mL})$ was refluxed on a water bath for 48 hrs. During reaction time hydrogen sulphide evolved. The solvent was removed by distillation under reduced pressure and the residue was crystallized from acetic acid as yellow cry stals of 8a-d (Tables 1. 2).

2-Methylthio-3,9-diphenyl-7-(2'-thienyl)-pyrido[3',2': 4,5]thieno[3,2-d]pyrimidne-4(3H)-one (9). Compound $8 \mathbf{a}$ (0.93 g. 2 mmol) was dissolved in $4 \%$ (10 mL. $10 \mathrm{mmol})$ ethanolic sodium hydroxide solution. To this solution methyl iodide $(0.2 \mathrm{~mL} .3 \mathrm{mmol})$ was added. The reaction mixture was refluxed for 2 lus. The precipitate was filtered off and recrsytallized from ethanol as white needles of 9 IR: $v=$ $1670(\mathrm{C}=\mathrm{O}) \mathrm{cm}^{-1}$. ${ }^{l} \mathrm{H}$ NMR (TFA.): $\delta=8.0-8.3(\mathrm{~m}, 3 \mathrm{H}$. $2 \mathrm{CH}$ thienyl and $\mathrm{CH}$ pyridine) $7.3-7.8$ (m. 11H: $10 \mathrm{ArH}$ 's and $\mathrm{CH}$ thienyl) 2.0 (s. $3 \mathrm{H} . \mathrm{SCH}_{\mathfrak{j}}$ ) ppm.

3,9-Diphenyl-2-phenacylthio-7-(2'-thienyl)-pyrido [3',2': 4,5]thieno[3,2-d]pyrimidine-4(3H)-one (10). To a suspension of compound $8 \mathrm{a}(0.94 \mathrm{~g} .2 \mathrm{mmol})$ and sodium acetate trihyddrate $(0.54 \mathrm{~g} .4 \mathrm{mmol})$ in ethanol $(20 \mathrm{~mL})$. phenacyl bromide ( $0.4 \mathrm{~g} .2 \mathrm{mmol}$ ) was added. The resulting mixture was refluxed for 2 hrs. The precipitate that formed while hot was collected and recrystallized from ethanol-chloroform mixture as yellow needles of 10. IR: $v=1660(\mathrm{C}=\mathrm{O}) \mathrm{cm}^{-1}$. ${ }^{l} \mathrm{H}$ NMR (T.F.A): $\delta=8.3-7.3$ (m, 19H, 15ArH's. 3CH thienyl and $\mathrm{CH}$ pyridine). 4.3 (s. $2 \mathrm{H} . \mathrm{SCH}_{2}$ ) ppm.

3,9-Diphenyl-2-hydrazino-7-(2'-thienyl)-pyrido [3',2':t,5]thieno[3,2-d]pyrimidine- $+(3 H)$-one (11). A mixture of $8 \mathbf{a}$ (1.9 g. $4 \mathrm{mmol}$ ) and hydrazine hydrate $99 \%$ (4.0 mL. 80 mmol) in pyridine $(20 \mathrm{~mL})$ was heated under reflux for 7 hrs. The product which precipitated while hot was collected and recrystallized from dioxane as pale yellow crystals of 11. IR: $v=3300.3200\left(\mathrm{NHNH}_{2}\right), 1670(\mathrm{C}=\mathrm{O}) \mathrm{cm}^{-1}$. MS: $467.97\left(\mathrm{M}^{+}, 10 \%\right), 376\left(\mathrm{M}^{-}-\mathrm{PhN}, 100 \%\right), 332.89\left(\mathrm{M}^{+}-\mathrm{PhN}-\right.$
CS. 38\%), $304\left(\mathrm{M}^{+}-\mathrm{PhN}-\mathrm{CS}-\mathrm{CO} .45 \%\right) .77\left(\mathrm{C}_{6} \mathrm{H}_{5}^{+} .90 \%\right)$. 4,10-Diphenyl-8-(2'-thienyl)-s-triazolo[4",3"-alpyrido$\left[3^{\prime}, 2^{\prime}: 4,5\right]$ thieno[3,2-e] pyrimidine-5 (4H)-one (12). A mixture of hydrazino compound $11(0.4 \mathrm{~g} .1 \mathrm{nmmol})$ and triethyl orthoformate $(7 \mathrm{~mL})$ was heated under reflux for 4 hrs. The precipitate that formed while hot was filtered off and recrytallized from DMF to give pale yellow crystals of 12. IR: $v=1670(\mathrm{C}=\mathrm{O}) \mathrm{cm}^{-1}$. ' $\mathrm{H}$ NMR (TFA): $\delta=9.3$ (s. 1H. CH triazole) $7.4-8.5$ (m. 14H: 10 ArH's. 3CH thienyl and $\mathrm{CH}$ pyridine). $\mathrm{MS}: 477\left(\mathrm{M}^{+}, 5 \%\right)$.

Biological screening. The filter paper disc method was performed in Nutrient agar for bacteria and Dox agar for fungi. These agar media were inoculated with $0.5 \mathrm{~mL}$ of the $24 \mathrm{~h}$. liquid cultures. Filter paper discs $(5 \mathrm{~mm}$ diameter) saturated with each compound solution $(10 \mathrm{mg} / \mathrm{mL}$ of DMSO) were placed on the indicated agar media. The incubation time was $48 \mathrm{~h}$ (at $37^{\circ} \mathrm{C}$ for bacteria and at $28^{\circ} \mathrm{C}$. for fungi). Discs saturated with DMSO were used as control. The diameter of inlubition zones $(\mathrm{mm})$ were measured and recorded.

\section{References}

1. Eichenberger, K: Schweizer. E: Schmidt, P. LIS. Patent 2.627.7661+. 1971: Chem. Abstr: 1971.74.886.38w:

2. Burger. A. Medicinal Chemisty. 3rd Ed.: Wiley-InterSciences: New York (N.Y.). 1970: Vol 72. p 544. p 719.

3. Shraideh, Z.: Sallal. A.-K. Bioned Lett. 1997.54, 233.

4. Gilis. P. M. Haemers. A.: Bollaert. W. Eur. J. Med Chem. 1980. 15. 185.

5. Bompart. T.: Giral. L.: Malicome. G.: Puygrenier. M. Eur J. Med. Chem. 1987. 22. 139 .

6. Adachi. I.: Hiramatsu. Y. Jap. Pat. 03.52.890. Chem. Abst: 1991. 115. 71573 .

7. Furuya. S.; Takeyru. N.; Matsumoto, H. Jap. Pat. 09.169,766; Chent. Abst: 1997. 127. 176416. 
8. Furuya, S: Choh. N.: Suzuki, N.: Imada. T. PCT Int Appt WO, 000.00.493: Chem. Abstr. 2000. 132.64179.

9. Dave. C. G.: Shah. P. R.: Dave. K. C.: Patel. V. J. J. Indian Chem. Soc. 1989.66. 48 .

10. Bousquent. E.: Romero. G.: Guemera. F; Carlso. A: Roxas, M. A. Farntaco Ed Sci. 1985, 40,869.

11. Leistner, S: Wagener, G.: Guestscharo, M: Glusa, E. Pham 1986. 41.54

12. Wagner. G.: Leistner. $\mathrm{S}$ : Vieweg. $\mathrm{H}$ : Krasselt. IJ: Prantz. J. Phamazie 1993. 49. 514.

13. Youssefyeh. R. D.: Brown. R. E: Wilson, J.; Shah, U.: Jones. H.: Loev, B.: Khandwala. A.: Leibowitz, M. J.: Sonnino-Goldman, P.
J. Med Chen $1984,27,1639$.

14. Bakhite. E. A.: Abdel-Rahman1. A. E.: Mohamed. O. S.: Thabet. E. A. Pharmazie 2000. 55.577.

15. Abdel-Rahmant. A. E.: Bakhite. E. A.: Mohamed. O. S.: Thabet. E. A. Phosphornis. Sulfur and Silicon 2000, 166. 149.

16. Abdel-Rahman. A. E: Baklite. E. A.; Al-Taiti. E. A. J. Chm Chent Soc. $2002,+9.223$.

17. Abdel-Rahman. A. E.; Bahihite. E. A.: Mohamed, O. S.; Thabet. E. A. Phosphortis. Sulfur and Silicon 2002 (acceptance).

18. Kalyoncuoglu. N.: Rollas. S.: Sur-Altiner. D.: Yegenoglu. Y.: Ang. O. Phamazie 1992. 47.769 . 